\title{
Plasmon-polariton emission from a coherently $p$-excited quantum dot near a metal interface
}

\author{
C. Sanchez-Munoz, A. Gonzalez-Tudela, ${ }^{*}$ and C. Tejedor \\ Física Teórica de la Materia Condensada, Universidad Autónoma de Madrid, 28049, Spain
}

(Received 29 September 2011; published 6 March 2012)

\begin{abstract}
We study the emission of surface plasmon polaritons by the decay of the lowest excited state of a quantum emitter when the system is excited by a laser in resonance with a higher excited state ( $p$-shell excitation). By solving a master equation and by using the quantum-regression theorem, we show how the emission is enhanced by the Purcell effect due to the weak coupling between the emitter and the structured spectral density of plasmon-polariton states of a metal surface. Measurable magnitudes, as the spectrum and the second-order coherence function, are extremely affected by the coherent $p$-shell excitation. In many cases, such coherent excitation completely masks the physical features of the emission under study. The coexistence between coherent $p$-shell excitation in the first step of the process and weak coupling in the final step is very important and completely general for any structured reservoir of final states. The advantage of our system is that, just by changing the distance from the quantum emitter to the metal surface, one can access a very rich set of regimes as purely dissipative direct photon emission or emission of plasmon polaritons.
\end{abstract}

DOI: 10.1103/PhysRevB.85.125301

PACS number(s): 42.50.Nn, 42.50.Ex, 73.20.Mf

\section{INTRODUCTION}

Photon emission produced by the decay of a two-level system can be manipulated by modifying the density of final photon states. This is the well-known Purcell effect ${ }^{1}$ extensively studied for quantum emitters (QEs) as, for instance, quantum dots, weakly coupled to the quasidiscrete photon spectrum of microcavities. ${ }^{2-4}$ One of the main difficulties for such kinds of experiments is related to the excitation of the QE, i.e., how to populate state $|1\rangle$ from state $|0\rangle$ shown in the sketch of Fig. 1. The easiest solutions are the photoexcitation ones at frequencies significantly higher than that of the transition of interest or the electron and hole electrical injection. But these alternatives imply energy relaxation usually by means of acoustic phonon emission. This incoherent pumping introduces decoherence and dephasing effects in the system, which, although interesting for some purposes ${ }^{5-7}$ mask the characteristics of the emission process itself. Therefore, coherent pumping is desirable in most cases. However, using pumping lasers with frequencies as close as possible to the transition to be studied introduces a series of practical difficulties. ${ }^{8,9}$ Therefore, a frequent alternative, usually known as a $p$-shell excitation, ${ }^{10,11}$ is to be excite resonantly to a third (higher) level of the QE labeled $|2\rangle$ in Fig. 1. Since transitions $|0\rangle \rightarrow|2\rangle$ and $|1\rangle \rightarrow|0\rangle$ must be electric dipole allowed, transition $|2\rangle \rightarrow|1\rangle$ is forbidden and must be assisted by one nonradiative excitation as, for instance, phonon emission. $p$-shell excitation involves coherent coupling between the laser field and the $|0\rangle \rightarrow|2\rangle$ transition, whereas, the Purcell effect involves weak coupling between the $|1\rangle \rightarrow|0\rangle$ transition and the emitted photon. The interplay between these coherent and incoherent regimes of coupling has consequences on the interference of emitted photons, which theoretically has been analyzed in cavity quantum electrodynamics. ${ }^{12,13}$ The aim of this paper is the study of similar effects on measurable magnitudes as populations, emission spectrum, and second-order coherence functions in a system with a continuous spectrum. So, instead of working with a microcavity that presents a density of states with a simple Lorentzian shape, we choose a system with a structured density of states: the surface plasmon-polariton (SPP) field of a planar metal-dielectric interface. ${ }^{14}$ This is the electromagnetic excitation of the system presenting plasmonic character inside the metallic region and photonic character outside. ${ }^{15}$ They allow subwavelength confinement of the electromagnetic field for the optical regime and have been proposed to be excellent candidates for building photonic circuits, ${ }^{16}$ coupling to QEs, ${ }^{17,18}$ showing quantum-interference effects, ${ }^{19,20}$ and even inducing entanglement between them. ${ }^{21}$ The main reason for our choice is that, just by moving the QE spatially with respect to the metal interface, one can access three different emission regimes: from purely dissipative for small separations to direct photon emission to the semi-infinite dielectric for very large separations passing through the most interesting regime of SPP emission at intermediate separations. This gives a great richness of situations not available in other systems.

The outline of the paper is as follows: Section II contains the theoretical study of the system dynamics by means of a master equation. The QE is described by a three-level system (see Fig. 1) coherently excited by a laser in resonance with transition $|0\rangle \rightarrow|2\rangle$. Nonradiative $(|2\rangle \rightarrow|1\rangle)$ and radiative $(|1\rangle \rightarrow|0\rangle)$ decays are described by Lindblad terms. The distance of the $\mathrm{QE}$ to the interface determines three different regimes of emission depending on the spectral density of the system, which is studied in this section by means of a Green's function formalism. Measurable magnitudes as populations, spectrum, and second-order coherence are calculated and are discussed in Sec. III with special attention to the regime of SPP emission. Finally, some conclusions are drawn in Sec. IV.

\section{THEORETICAL DESCRIPTION}

We study the dynamics of a metal-dielectric interface coupled to a QE excited by a laser by means of a master equation. By tracing out in the degrees of freedom of the electromagnetic excitations of the interface, one gets a master equation for the $\mathrm{QE}$ density matrix only. Moreover, we work within the usual Born-Markov and rotating-wave approximations. ${ }^{22}$ 

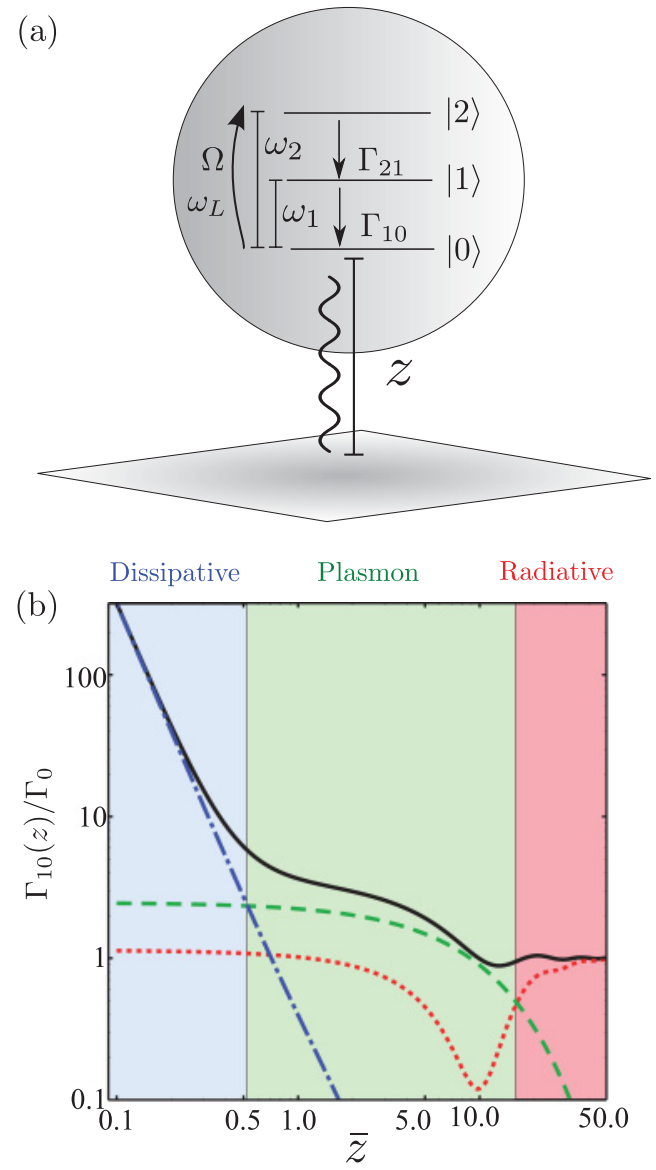

FIG. 1. (Color online) (a) Schematic view of a three levels quantum emitter, with characteristic frequencies $\omega_{1}$ and $\omega_{2}$, placed at a distance $z$ of an infinite planar metallic surface. (b) $\Gamma_{10}$ (black continuous line) as a function of $\bar{z}$. The three contributions to $\Gamma_{10}$ are depicted by a dashed-dotted blue line for dissipation, a dashed green line for plasmons and a dotted red line for radiation.

\section{A. Master equation}

The coherent part of the dynamics is described by a Hamiltonian of the three-level system $\{|0\rangle,|1\rangle,|2\rangle\}$ excited by a laser treated as a coherent field of frequency $\omega_{L}(\hbar=1$ throughout the paper),

$$
H=\omega_{1} \hat{\sigma}_{11}+\omega_{2} \hat{\sigma}_{22}+\Omega\left(\hat{\sigma}_{02} e^{+i \omega_{L} t}+\hat{\sigma}_{20} e^{-i \omega_{L} t}\right),
$$

where the ground state has been taken as the origin of energies and $\hat{\sigma}_{i j}=|i\rangle\langle j|$. Using a unitary transformation $U=e^{i \omega_{L} t \hat{\sigma}_{22}}$, the Hamiltonian transforms into

$$
H=\omega_{1} \hat{\sigma}_{11}+\delta_{2} \hat{\sigma}_{22}+\Omega\left(\hat{\sigma}_{02}+\hat{\sigma}_{20}\right),
$$

where $\delta_{2}=\omega_{2}-\omega_{L}$.

Apart from the coherent excitation from the ground state to the upper level $|2\rangle$, the dynamics of this open system includes the cascade decays $|2\rangle \rightarrow|1\rangle$ and $|1\rangle \rightarrow|0\rangle$ described by Lindblad terms,

$$
\begin{aligned}
& \mathcal{L}_{1} \rho=\frac{\Gamma_{10}}{2}\left(2 \hat{\sigma}_{01} \rho \hat{\sigma}_{10}-\rho \hat{\sigma}_{11}-\hat{\sigma}_{11} \rho\right), \\
& \mathcal{L}_{2} \rho=\frac{\Gamma_{21}}{2}\left(2 \hat{\sigma}_{12} \rho \hat{\sigma}_{21}-\rho \hat{\sigma}_{22}-\hat{\sigma}_{22} \rho\right),
\end{aligned}
$$

in a master equation $\dot{\rho}=i[\rho, H]+\mathcal{L}_{1} \rho+\mathcal{L}_{2} \rho$. The coexistence of coherent pumping and incoherent decays brings the system to a stationary regime.

This description of the dynamics is adequate for experiments with a coherent excitation of state $|2\rangle$ strong enough to have excitation intensity $\Omega$ larger than or comparable to $\Gamma_{21}$. However, if the decay from $|2\rangle$ to $|1\rangle$ is so fast that $\Gamma_{21} \gg \Omega$ and $\Gamma_{21} \gg \delta_{2}$, then, state $|2\rangle$ can be eliminated adiabatically from the dynamics, and the problem becomes that of an incoherent pumping of level $|1\rangle$ described by a Lindblad-like term,

$$
\mathcal{L}_{P} \rho=\frac{P_{01}}{2}\left(2 \hat{\sigma}_{10} \rho \hat{\sigma}_{01}-\rho \hat{\sigma}_{00}-\hat{\sigma}_{00} \rho\right),
$$

with an effective rate,

$$
P_{01}=\frac{4 \Omega^{2} \Gamma_{21}}{\Gamma_{21}^{2}+4 \delta_{2}^{2}} .
$$

The nonradiative rate transition $\Gamma_{21}$ does not depend on the coupling between the QE and the SPP. Therefore, it will be taken just as a constant parameter. The radiative decay with rate $\Gamma_{10}$ is much higher as a consequence of the Purcell effect coming from the coupling of transition $|1\rangle \rightarrow|0\rangle$ to the modes of a metal-semiconductor interface. Therefore, it requires a careful analysis in the next subsection.

\section{B. Spectral density and modified lifetime}

The physics behind transition $|1\rangle \rightarrow|0\rangle$ is that of an oscillating dipole coupled to the excitations of a dissipative metal-dielectric interface as depicted in Fig. 1. The QE in the upper-half space is embedded within a dielectric matrix with a dielectric function that can be taken in the range of frequencies of interest as real and constant $\epsilon_{1}$. In the lower-half space $z<0$, a dissipative metal is characterized by a complex dielectric function $\epsilon_{2}(\omega)$. In order to properly describe the dissipation in the metal, we use a dielectric function, ${ }^{23}$

$\epsilon_{2}(\omega)=\epsilon_{r}-\sum_{j} \frac{\omega_{P j}^{2}}{\omega\left(\omega+i \eta_{j}\right)}-\sum_{j} \frac{\Delta \epsilon_{j} \Omega_{j}^{2}}{\omega^{2}-\omega_{j}^{2}+i \omega \Upsilon_{j}}$,

with only one term $(j=0)$ in the sums.

The metal we consider for our calculations is silver for which the different parameters in Eq. (6) are $\epsilon_{r}=4.6, \omega_{P 0}=$ 9.0, $\eta_{0}=0.07, \Delta \epsilon_{0}=1.10, \Upsilon_{0}=1.2 \mathrm{eV}$, and $\Omega_{0}=4.9$.

The electrodynamics of this layered geometry is described by the Green's tensor $\widehat{\mathbf{G}}\left(\mathbf{r}, \mathbf{r}^{\prime}, \omega\right)$. The dissipative dynamics of the oscillating dipole is contained in the local Green's tensor $\widehat{\mathbf{G}}\left(\mathbf{r}_{Q}, \mathbf{r}_{Q}, \omega\right)$, where $\mathbf{r}_{Q}$ denotes the QE location. This Green's tensor splits in two contributions: $\widehat{\mathbf{G}}\left(\mathbf{r}_{Q}, \mathbf{r}_{Q}, \omega\right)=$ $\widehat{\mathbf{G}}_{0}\left(\mathbf{r}_{Q}, \mathbf{r}_{Q}, \omega\right)+\widehat{\mathbf{G}}_{R}\left(\mathbf{r}_{Q}, \mathbf{r}_{Q}, \omega\right)$. The former is a direct contribution in the dielectric, whereas, the reflection contribution comes from the interaction of the dipole with the SPP. ${ }^{24,25}$ In this paper, we only consider the dipole oriented perpendicular to the the interface so that the only relevant term of the tensor is the $z z$ component, ${ }^{24,25}$

$$
\begin{aligned}
G_{R, z z}\left(\mathbf{r}_{Q}, \omega\right)= & -\frac{c^{2}}{4 \pi \omega^{2}} \int_{0}^{\infty} d q \frac{q^{3}}{\kappa_{1}(q, \omega)} \\
& \times\left[\frac{\epsilon_{1} \kappa_{2}(q, \omega)-\epsilon_{2}(\omega) \kappa_{1}(q, \omega)}{\epsilon_{1} \kappa_{2}(q, \omega)+\epsilon_{2}(\omega) \kappa_{1}(q, \omega)}\right] e^{-2 \kappa_{1}(q, \omega) z}
\end{aligned}
$$


where $\kappa_{i}(q, \omega)=\sqrt{\epsilon_{i}(\omega)(\omega / c)^{2}-q^{2}}$ for $i=1,2$. Hereafter, we work with the dimensionless magnitudes $\bar{z}=z \omega_{p} / c, \bar{q}=$ $q c / \omega_{p}$ and $\bar{\omega}=\omega / \omega_{p}$ where $\omega_{p}$ is the bulk plasmon frequency and $c$ is the speed of light.

The action of the absorbing medium on the QE, is described completely by the spectral density,

$$
J(\omega)=\frac{1}{\pi \epsilon_{0}} \mathbf{p} \cdot\left[\frac{\omega^{2}}{c^{2}} \operatorname{Im}\left[\widehat{\mathbf{G}}\left(\mathbf{r}_{Q}, \mathbf{r}_{Q}, \omega\right)\right]\right] \cdot \mathbf{p},
$$

which is, apart from constants, the tensor contraction of the local (at $\mathbf{r}_{Q}$ ) Green's function with the dipole moment $\mathbf{p}$ associated with the $|1\rangle \rightarrow|0\rangle$ transition. This spectral density also can be written as $J(\omega)=g^{2}(\omega) \rho(\omega)$ where $g(\omega)$ is the QE-environment coupling and $\rho(\omega)$ is the density of states of the environment. For a QE with a dipole oriented along the $z$ direction and a frequency splitting $\omega_{0}$ below the singularity at the SPP edge $\omega_{s p}$, the spectral density gives the modified lifetime in the Markov approximation [Eq. (3)] $\Gamma_{10}\left(\omega_{0}\right)=2 \pi J\left(\omega_{0}\right)$. Figure $1(\mathrm{~b})$ shows $\Gamma_{10}$, normalized to its vacuum value $\Gamma_{0}$, as a function of the renormalized separation $\bar{z}$ for an interface between the silver and a dielectric with $\epsilon_{1}=1$. There are three different regions: In the region of small separation $(\bar{z}<1 / 2), \Gamma_{10}$ varies as $(\bar{z})^{-3}$ because the dynamics is controlled by the dissipative part of the metal dielectric function. ${ }^{25}$ For large separations $(\bar{z}>50), \Gamma_{10}$ roughly is constant (just small oscillations) because it is mainly due to the direct radiative term $\widehat{\mathbf{G}}_{0}$. The intermediate region $(1 / 2<\bar{z}<50)$ is the most interesting one because it presents $\Gamma_{10}$ slowly decreasing with $\bar{z}$ as dominated by the emission of SPP. $^{26}$ This is the region in which we concentrate throughout this paper.

\section{POPULATIONS AND SECOND-ORDER CORRELATION FUNCTIONS}

Once all the ingredients of the master equation are known, one can calculate the dynamics density matrix and, in particular, the stationary state. By means of standard procedures in quantum optics, ${ }^{27}$ as the quantum-regression theorem, one computes the experimentally accessible magnitudes as level occupations, emission spectrum, and second-order coherence.

\section{A. Populations}

The simplest magnitudes to calculate are the populations of the levels that are obtained as expectation values of the $\hat{\sigma}$ operators $\left\langle\hat{\sigma}_{i j}\right\rangle=\operatorname{Tr}\left(\rho \hat{\sigma}_{i j}\right)=\rho_{j i}$. In other words, instead of the master equation for operators, one works with some kind of optical Bloch equations for the matrix elements,

$$
\begin{aligned}
& \dot{\rho}_{00}=\Gamma_{10} \rho_{11}+i \Omega\left(\rho_{02}-\rho_{20}\right), \\
& \dot{\rho}_{11}=\Gamma_{21} \rho_{22}-\Gamma_{10} \rho_{11}, \\
& \dot{\rho}_{22}=-\Gamma_{21} \rho_{22}-i \Omega\left(\rho_{02}-\rho_{20}\right), \\
& \dot{\rho}_{20}=-\left(i \delta_{2}+\frac{\Gamma_{21}}{2}\right) \rho_{20}+i \Omega\left(\rho_{22}-\rho_{00}\right), \\
& \dot{\rho}_{02}=\left(i \delta_{2}-\frac{\Gamma_{21}}{2}\right) \rho_{02}-i \Omega\left(\rho_{22}-\rho_{00}\right) .
\end{aligned}
$$
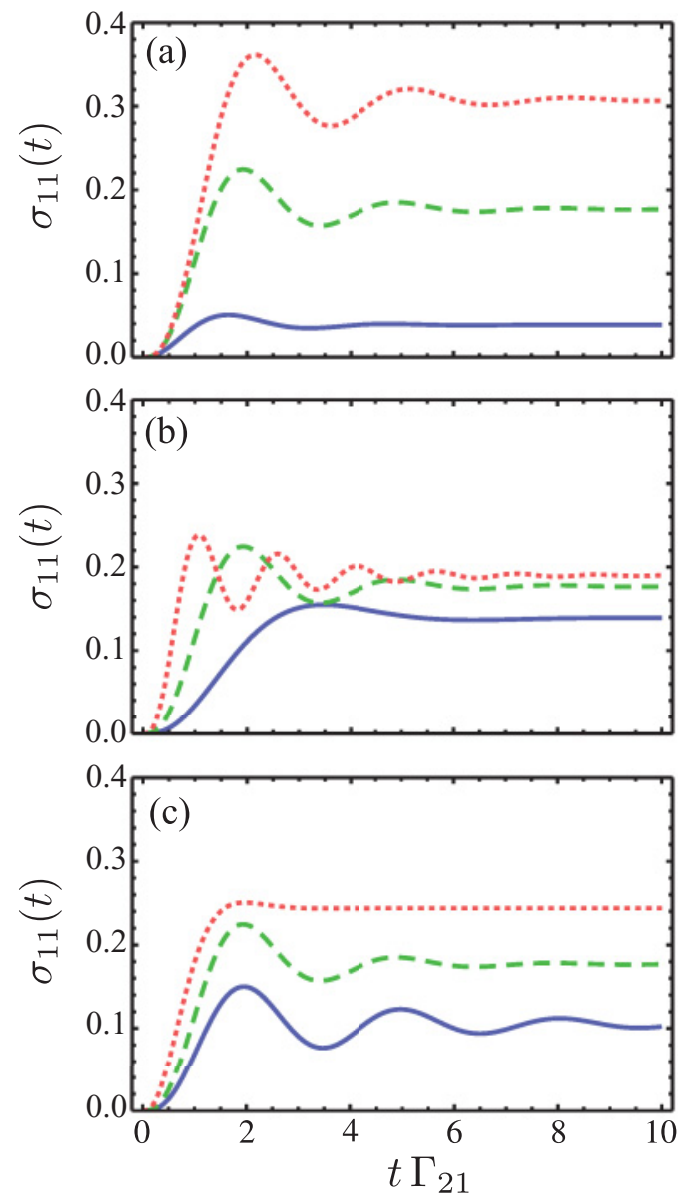

FIG. 2. (Color online) (a) Evolution of the population of the second level, $\sigma_{11}(t)$, for $\bar{z}=0.35$ (continuous blue line), 4.5 (dashed green line) and 50 (dotted red line). $\Gamma_{0}=\Gamma_{21}, \Omega=\Gamma_{21}$, and $\delta_{2}=0$. (b) Evolution of the population of the second level, $\sigma_{11}(t)$, for $\Omega=0.5 \Gamma_{21}$ (continuous blue line), $\Gamma_{21}$ (dashed green line) and $2 \Gamma_{21}$ (dotted red line). (c) Evolution of the population of the second level, $\sigma_{11}(t)$, for an increasing rate $\Gamma_{21} . \Gamma_{21} / \Omega=0.5$ (continuous blue line), $\Gamma_{21} / \Omega=1$ (dashed green line), and $\Gamma_{21} / \Omega=4$ (dotted red line). $\Omega=\Gamma_{0}=1, \delta_{2}=0$ and $\bar{z}=4.5$. A typical value of the non radiative decay time is $\Gamma_{21}^{-1}=10$ ps. ${ }^{13,28}$

Since $\rho_{00}+\rho_{11}+\rho_{22}=1$, Eq. (9) can be written as a vector equation,

$$
\frac{d \vec{u}}{d t}=M \vec{u}+\vec{P}
$$

where $\vec{u} \equiv\left(\rho_{00}, \rho_{11}, \rho_{20}, \rho_{02}\right)^{t}$ and $M$ is the $4 \times 4$ matrix given by Eq. (9). Steady-state populations are obtained by setting $\dot{\vec{u}}=0$,

$$
\rho_{11}^{s s}=\frac{4 \Gamma_{21} \Omega^{2}}{\Gamma_{10}\left(\Gamma_{21}^{2}+4 \delta_{2}^{2}\right)+4\left(2 \Gamma_{10}+\Gamma_{21}\right) \Omega^{2}}=\frac{\Gamma_{21}}{\Gamma_{10}} \rho_{22}^{s s} .
$$

Figure 2 shows the transient evolution of the population of state $|1\rangle$ for different separations for (a) a fixed laser intensity and for different values of the laser intensity for (b) a fixed separation. The three separations of panel (a) correspond to the center (green) and the two borders (blue and red) of the intermediate region where the emission of SPP dominates the electrodynamics of the system. Since a typical value of the 
nonradiative decay time is $\Gamma_{21}^{-1}=10 \mathrm{ps},{ }^{13,28}$ the transient time for reaching the steady state is less than $100 \mathrm{ps}$.

The physical origin of the oscillations has nothing to do with the coupling of the QE excitations to the SPP. It is a manifestation of the coherence of the excitation by the laser. This assertion can be checked from the results in panel (c) of the figure where $\Gamma_{12}$ is increased, whereas, the intensity $\Omega$ of the pumping is kept constant with respect to any other parameter. Then, the coherence associated with the excitation becomes spoiled by the fast nonradiative transition, and this manifests in a monotonic population evolution into its steady state. As aforementioned, the physics of the system in this regime may be mapped exactly into the two-level system $\{|0\rangle,|1\rangle\}$ incoherently pumped with a rate $P_{01}$ given by Eq. (5).

\section{B. Steady-state spectra}

The steady-state spectra are computed by using the WienerKhintchine formula,

$$
S^{s S}(\omega)=\frac{1}{\pi} \frac{1}{\rho_{11}^{s S}} \operatorname{Re} \int_{0}^{\infty} G^{(1)}(\tau) e^{i \omega \tau} d \tau,
$$

where $G^{(1)}(\tau)$ is the first-order correlation function,

$$
G^{(1)}(\tau)=\lim _{t \rightarrow \infty}\left\langle\hat{\sigma}_{10}(t) \hat{\sigma}_{01}(t+\tau)\right\rangle .
$$

This requires the calculation of a two-time correlation function, which can be performed by means of the quantumregression theorem. ${ }^{22,27}$ On top of Eq. (9), the initial conditions for the dynamics in the unknown $\tau$ require knowing the dynamics of two more elements, something also obtained from the master equation,

$$
\begin{aligned}
& \dot{\rho}_{10}=-\left(i \omega_{1}+\frac{\Gamma_{10}}{2}\right) \rho_{10}+i \Omega \rho_{12}, \\
& \dot{\rho}_{12}=i \Omega \rho_{10}+\left\{i\left(\delta_{2}-\omega_{1}\right)-\frac{\Gamma_{10}+\Gamma_{21}}{2}\right\} \rho_{12},
\end{aligned}
$$

which can be written as

$$
\frac{d \vec{w}}{d \tau}=N \vec{w},
$$

with $\vec{w} \equiv\left(\sigma_{01}, \sigma_{21}\right)^{t}$ and $N$ as the $2 \times 2$ matrix given Eq. (14). The quantum-regression theorem allows calculating $G^{(1)}(\tau)$ by solving

$$
\frac{d \vec{p}}{d \tau}=N \vec{p}
$$

where now $\vec{p}$ is given by $\vec{p} \equiv\left(\left\langle\hat{\sigma}_{10}(t) \hat{\sigma}_{01}(t+\right.\right.$ $\left.\tau)\rangle,\left\langle\hat{\sigma}_{10}(t) \hat{\sigma}_{21}(t+\tau)\right\rangle\right)^{t}$ and the initial conditions are given by $\vec{p}(0)=\left(\rho_{11}^{s s}, 0\right)^{t}$. The formal solution of Eq. (16) is $\vec{p}=\vec{p}(0) e^{N \tau}$, whose first component is taken to calculate $G^{(1)}(\tau)$ and $S^{s s}(\omega)$, which finally takes the form

$$
S^{s S}(\omega)=\frac{1}{\pi} \operatorname{Re} \int_{0}^{\infty}\left(e^{N \tau}\right)_{1,1} e^{i \omega \tau} d \tau=\frac{2}{\pi} \operatorname{Re} \frac{\Gamma_{10}+\Gamma_{21}+2 i\left(\omega_{1}-\delta_{2}-\omega\right)}{4 \Omega^{2}+\left[\Gamma_{10}+2 i\left(\omega_{1}-\omega\right)\right]\left[\Gamma_{10}+\Gamma_{21}+2 i\left(\omega_{1}-\delta_{2}-\omega\right)\right]}
$$

Figure 3 shows the spectra of the emission produced by transition $|1\rangle \rightarrow|0\rangle$. Panels (a) and (b) are computed under the same conditions as the populations given in Fig. 2, whereas, panel (c) depicts the case with finite detuning $\delta_{2}$. The calculations have been performed by taking an energy difference between the two excited levels $\omega_{21}=\omega_{2}-\omega_{1}=$ $23.6 \mathrm{meV}$, which is a typical value for InAs/GaAs quantum dots. ${ }^{29}$ One clearly can observe how the incoherence associated with transition $|1\rangle \rightarrow|0\rangle$ tends to hide any feature associated with the $|0\rangle \rightarrow|2\rangle$ and $|2\rangle \rightarrow|1\rangle$ transitions. However, when the intensity $(\Omega)$ of the exciting laser is high compared to the decay rate $\left(\Gamma_{21}\right)$ of level $|2\rangle$, it produces a coherent excitation of the $|0\rangle \rightarrow|2\rangle$ transition that can be detected as a splitting in the spectrum of $|1\rangle \rightarrow|0\rangle$ emission. This competition of effects can be observed in panel (a) of the figure where the pumping intensity is fixed $\left(\Omega=\Gamma_{21}\right)$ and one can observe that the spectrum shows a single peak for small values of $\bar{z}$ (large $\Gamma_{10}$ 's). By increasing $\bar{z}$, the spectrum evolves to a double-peak structure, which becomes clearly resolved in this radiative regime. Another way of observing the same effects is shown in panel (b) where $\bar{z}$ is fixed within the regime of SPP emission. Again, one can observe the evolution from a single-peak to a double-peak structure just by increasing the pumping intensity $\Omega$. Finally, in panel (c), we show the emission spectrum for different values of the laser detuning $\delta_{2}$, a parameter easy to control experimentally. The spectrum becomes asymmetric when the laser is detuned slightly with respect to the $|0\rangle \rightarrow|2\rangle$ transition, i.e., $\delta_{2} \neq 0$. The interest in which this effect resides should clearly be observable.

\section{Second-order correlation function}

Further insight into the characteristics of the emission, as the possibility of single SPP emission measurable in a Hanbury Brown-Twiss setup, ${ }^{30}$ is contained in the secondorder coherence function,

$$
g^{(2)}(\tau)=\lim _{t \rightarrow \infty} \frac{\left\langle\hat{\sigma}_{10}(t) \hat{\sigma}_{10}(t+\tau) \hat{\sigma}_{01}(t+\tau) \hat{\sigma}_{01}(t)\right\rangle}{\rho_{11}^{s s} \rho_{11}^{s s}} .
$$

The technique for computing this two-time magnitude again resides in the use of the quantum-regression theorem with vector $\vec{v}(t, \tau) \equiv\left(\left\langle\hat{\sigma}_{10}(t) \hat{\sigma}_{00}(t+\tau) \hat{\sigma}_{01}(t)\right\rangle,\left\langle\hat{\sigma}_{10}(t) \hat{\sigma}_{11}(t+\right.\right.$ $\left.\left.\tau) \hat{\sigma}_{01}(t)\right\rangle,\left\langle\hat{\sigma}_{10}(t) \hat{\sigma}_{02}(t+\tau) \hat{\sigma}_{01}(t)\right\rangle,\left\langle\hat{\sigma}_{10}(t) \hat{\sigma}_{20}(t+\tau) \hat{\sigma}_{01}(t)\right\rangle\right)^{t}$.

Following a procedure similar to the one described above for $G^{(1)}(\tau)$, one gets the set of equations,

$$
\frac{d \vec{v}}{d \tau}=M \vec{v}+\rho_{11}^{s s} P
$$

to be solved with initial conditions $\vec{v}(0) \equiv\left(\rho_{11}^{s s}, 0,0,0\right)^{t}$. A particular solution is that for a steady state in which $\dot{\vec{v}}=0$. Then, the set of equations is similar to the one obtained for $\vec{u}$ except for the independent term, which, instead of being 


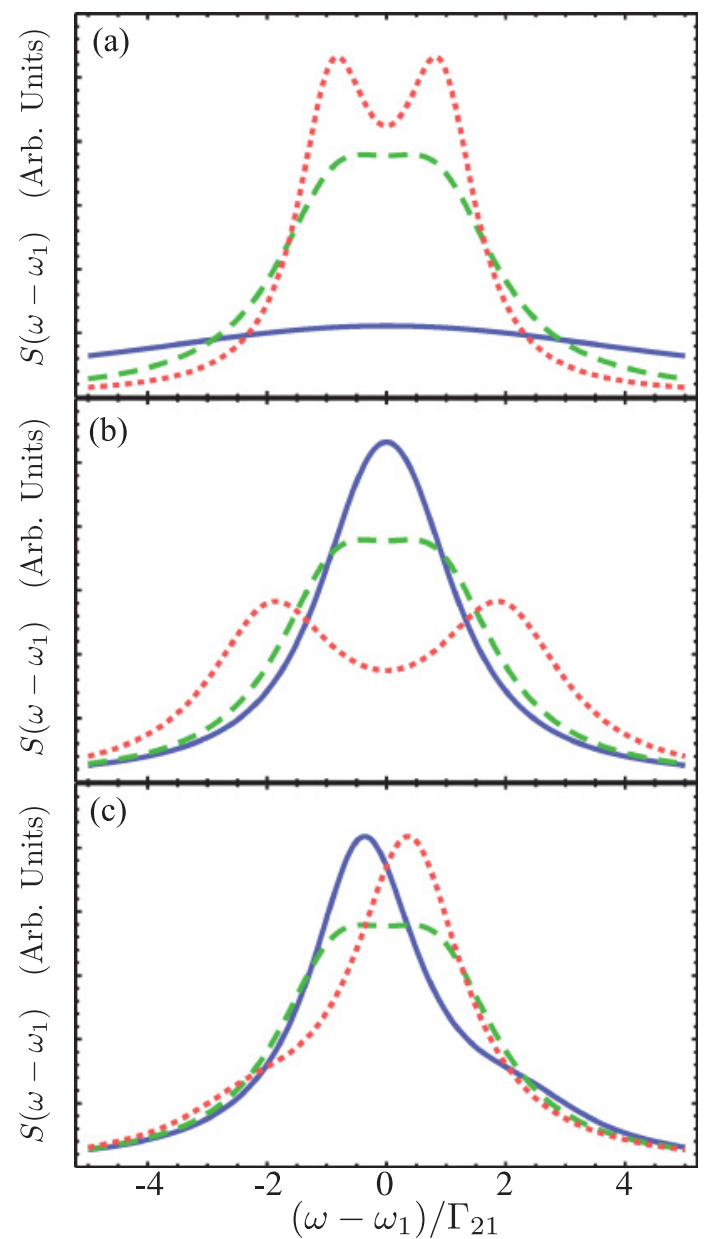

FIG. 3. (Color online) (a) Steady-state spectra $S(\omega)$ with $\bar{z}=0.35$ (continuous blue line), 4.5 (dashed green line) and 50 (dotted red line). $\Gamma_{0}=\Gamma_{21}$, and $\Omega=\Gamma_{21}, \delta_{2}=0$. (b) Steady-state spectra $S(\omega)$, for $\Omega=0.5 \Gamma_{21}$ (continuous blue line), $\Gamma_{21}$ (dashed green line) and $2 \Gamma_{21}$ (dotted red line). $\Gamma_{0}=\Gamma_{21}, \delta_{2}=0$ and $\bar{z}=4.5$. (c) Steady-state spectra $S(\omega)$, for $\delta_{2}=-2 \Gamma_{21}$ (continuous blue line), 0 (dashed green line), and $2 \Gamma_{21}$ (dotted red line). $\Gamma_{0}=\Gamma_{21}, \Omega=\Gamma_{21}$, and $\bar{z}=4.5$. A typical value of the non-radiative decay time of $10 \mathrm{ps}^{13,28}$ corresponds to an energy scale $\Gamma_{21}=400 \mu \mathrm{eV}$.

$\vec{P}$, is $\rho_{11}^{s s} \vec{P}$. So, this particular steady-state solution is just $\vec{v}^{s s}=\rho_{11}^{s s} \vec{u}^{s s}$. In order to have a general solution of Eq. (19), one needs the solution of the homogeneous equation, which is $\vec{v}^{\text {hom }}=e^{M \tau} \vec{v}(0)$. So, the general solution of Eq. (19) becomes $\vec{v}(\tau)=e^{M \tau} \vec{f}+\rho_{11}^{s s} \vec{u}^{s s}$, where $\vec{f}=\vec{v}(0)-\rho_{11}^{s s} \vec{u}^{s s}$. From the second component of vector $\vec{v}$, one gets

$$
g^{(2)}(\tau)=1-\frac{1}{\left(\rho_{11}^{s s}\right)^{2}}\left(e^{M \tau} \cdot \vec{f}\right)_{2} .
$$

$g^{(2)}(\tau)$ for transition $|1\rangle \rightarrow|0\rangle$ is shown in Fig. 4. The conditions are those of panels (a) and (b) in Fig. 3. When separation $\bar{z}$ is varied, the oscillatory behavior of $g^{(2)}(\tau)$ does not change much as shown in panel (a) of the figure. This proves that, in these conditions, transition $|0\rangle \rightarrow|2\rangle$ always is produced incoherently by the laser field even if, in the spectrum [panel (a) of Fig. 3], one observes a single peak. The interesting consequence is that decoherence $\Gamma_{10}$ hides the resolution of the double-peak structure of the spectrum, but the coherent
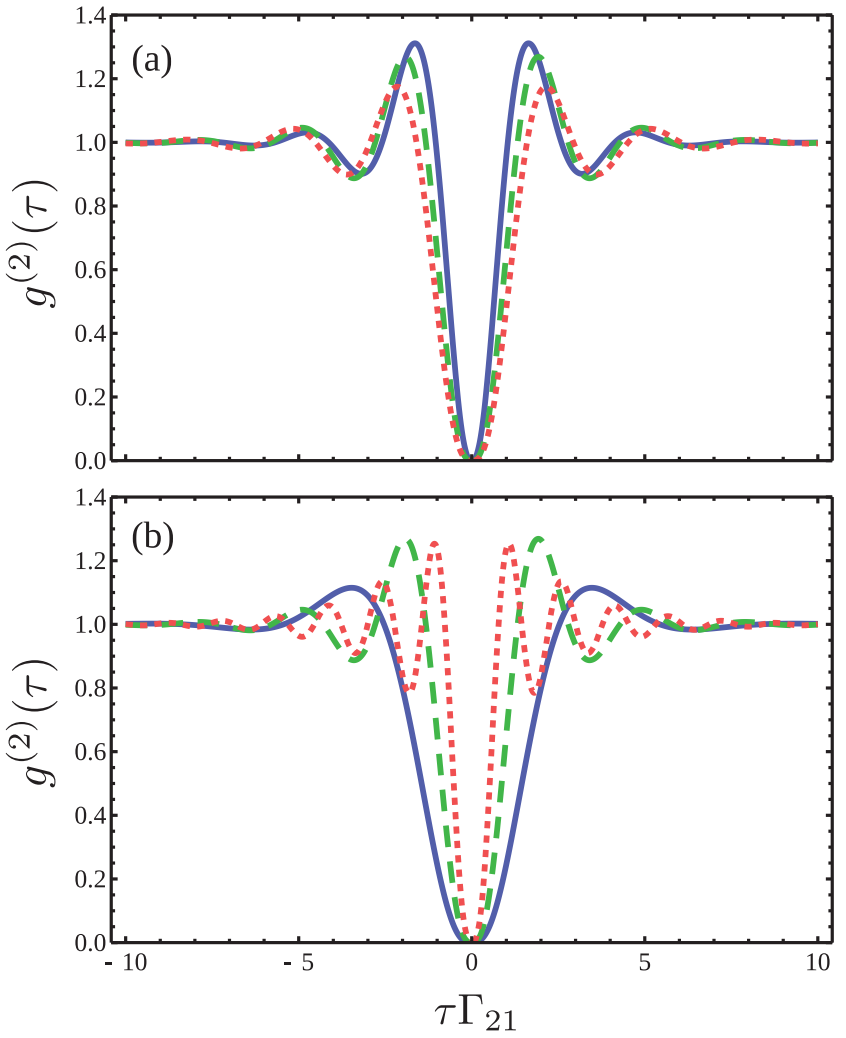

FIG. 4. (Color online) (a) Second order correlation function $g^{(2)}(\tau)$, for $\bar{z}=0.35$ (continuous blue line), 4.5 (dashed green line) and 50 (dotted red line). $\Gamma_{21}=1, \Omega=\Gamma_{21}$, and $\delta_{2}=0$. (b) Second order correlation function $g^{(2)}(\tau)$, for $\Omega=0.5 \Gamma_{21}$ (continuous blue line), $\Gamma_{21}$ (dashed green line) and $2 \Gamma_{21}$ (dotted red line). $\Gamma_{10}=$ $\Gamma_{21}, \delta_{2}=0$ and $\bar{z}=4.5$. A typical value of the non radiative decay time is $\Gamma_{21}^{-1}=10$ ps. $^{13,28}$

excitation $|0\rangle \rightarrow|2\rangle$ by the laser remains observable in photonphoton correlation spectroscopy [the Hanbury Brown-Twiss ${ }^{30}$ experiment measuring $\left.g^{(2)}(\tau)\right]$. A stronger effect in $g^{(2)}(\tau)$ is obtained by changing the intensity of the exciting laser. The oscillations, existing for high excitation, are quenched by decreasing the pumping up to the point in which just only a broad antibunching exists. As in the case of the spectrum, high laser intensity produces a coherent excitation of the $|0\rangle \rightarrow$ |2) transition, which dominates the emission characteristics producing oscillations in $g^{(2)}(\tau)$. However, for low-intensity excitation, the weak coupling between the $|1\rangle \rightarrow|0\rangle$ transition and the SPP dominates, and no oscillations exist anymore.

\section{CONCLUSIONS}

The main conclusion to be drawn from our paper is that one must be very careful when analyzing the photon or SPP emission from a QE under $p$-shell excitation. The Purcell effect producing the desired emission may coexist with a coherent excitation of the $p$-shell transition. In many cases, this coherent excitation produces oscillations that mask the physical characteristics of the final emission of interest. This important point, not commonly taken into account when analyzing experiments, is completely general for any structured reservoir of final states. In order to draw this 
conclusion, we have not worked with the simple case of a QE coupled to the optical modes of a microcavity having a density of states with a simple Lorentzian shape. Instead, we choose a system with a structured density of states: the SPP field of a planar metal-dielectric interface. ${ }^{14}$ This electromagnetic excitation presents plasmonic character inside the metallic region and photonic character outside. ${ }^{15}$ The main reason for our choice is that, just by changing the distance of the QE to the metal interface, one can access three different emission regimes: from purely dissipative for small separations to direct photon emission to the semi-infinite dielectric for very large separations passing through the most interesting regime of SPP emission at intermediate separations. This gives a very rich set of regimes not available in other systems.

\section{ACKNOWLEDGMENTS}

This work was supported by the Spanish MICINN under Contracts No. MAT2008-01555 and No. MAT2011-22997 and by CAM under Contract No. S-2009/ESP-1503. C.S.-M. acknowledges a grant from the Universidad Autonoma de Madrid. A.G.-T. acknowledges an FPU Grant No. AP200800101 from the Spanish Ministry of Education. *alejandro.gonzalez@uam.es

${ }^{1}$ E. M. Purcell, H. C. Torrey, and R. V. Pound, Phys. Rev. 69, 37 (1946).

${ }^{2}$ J. M. Gérard, B. Sermage, B. Gayral, B. Legrand, E. Costard, and V. Thierry-Mieg, Phys. Rev. Lett. 81, 1110 (1998).

${ }^{3}$ P. Michler, A. Kiraz, C. Becher, W. Schoenfeld, P. Petroff, L. Zhang, E. Hu, and A. Imamoglu, Science 290, 2282 (2000).

${ }^{4}$ C. Santori, D. Fattal, J. Vuckovic, G. S. Solomon, and Y. Yamamoto, Nature (London) 419, 594 (2002).

${ }^{5}$ F. P. Laussy, E. del Valle, and C. Tejedor, Phys. Rev. Lett. 101, 083601 (2008).

${ }^{6}$ F. P. Laussy, E. del Valle, and C. Tejedor, Phys. Rev. B 79, 235325 (2009).

${ }^{7}$ E. del Valle, F. P. Laussy, and C. Tejedor, Phys. Rev. B 79, 235326 (2009).

${ }^{8}$ A. Muller, E. B. Flagg, P. Bianucci, X. Y. Wang, D. G. Deppe, W. Ma, J. Zhang, G. J. Salamo, M. Xiao, and C. K. Shih, Phys. Rev. Lett. 99, 187402 (2007).

${ }^{9}$ E. B. Flagg, A. Muller, J. Robertson, S. Founta, D. G. Deppe, G. J. S. M. Xiao, W. Ma, and C. K. Shih, Nat. Phys. 5 (2009).

${ }^{10}$ S. Ates, S. M. Ulrich, S. Reitzenstein, A. Löffler, A. Forchel, and P. Michler, Phys. Rev. Lett. 103, 167402 (2009).

${ }^{11}$ P. Michler, Single Semiconductor Quantum Dots (Springer-Verlag, Berlin, 2009).

${ }^{12}$ A. Kiraz, M. Atatüre, and A. Imamoğlu, Phys. Rev. A 69, 032305 (2004).

${ }^{13}$ F. Troiani, J. I. Perea, and C. Tejedor, Phys. Rev. B 73, 035316 (2006).

${ }^{14}$ A. Gonzalez-Tudela, F. J. Rodríguez, L. Quiroga, and C. Tejedor, Phys. Rev. B 82, 115334 (2010).

${ }^{15}$ H. Raether, Surface Plasmons (Springer-Verlag, Berlin, 1988).

${ }^{16}$ T. W. Ebbesen, C. Genet, and S. I. Bozhevolnyi, Phys. Today 61(5), 44 (2008).
${ }^{17}$ A. V. Akimov, A. Mukherjee, C. L. Yu, D. E. Chang, A. S. Zibrov, P. R. Hemmer, H. Park, and M. D. Lukin, Nature (London) 450, 402 (2007).

${ }^{18}$ A. Trügler and U. Hohenester, Phys. Rev. B 77, 115403 (2008).

${ }^{19}$ V. Yannopapas, E. Paspalakis, and N. V. Vitanov, Phys. Rev. Lett. 103, 063602 (2009).

${ }^{20}$ S. Evangelou, V. Yannopapas, and E. Paspalakis, Phys. Rev. A 83, 055805 (2011).

${ }^{21}$ A. Gonzalez-Tudela, D. Martin-Cano, E. Moreno, L. MartinMoreno, C. Tejedor, and F. J. Garcia-Vidal, Phys. Rev. Lett. 106, 020501 (2011).

${ }^{22}$ H.-P. Breuer and F. Petruccione, The Theory of Open Quantum Systems (Oxford University Press, Oxford, 2002).

${ }^{23}$ S. G. Rodrigo, F. J. García-Vidal, and L. Martín-Moreno, Phys. Rev. B 77, 075401 (2008).

${ }^{24}$ S. Scheel and S. Y. Buhmann, Acta Physica Slovaca 58, 675 (2008).

${ }^{25}$ L. Novotny and B. Hecht, Principles of Nano-Optics (Cambridge University Press, Cambridge, UK, 2006).

${ }^{26}$ W. Barnes, J. Mod. Opt. 45, 661 (1998).

${ }^{27}$ Y. Yamamoto and A. İmamoḡlu, Mesoscopic Quantum Optics (Wiley, Hoboken, NJ, 1999).

${ }^{28}$ E. A. Zibik, L. R. Wilson, R. P. Green, G. Bastard, R. Ferreira, P. J. Phillips, D. A. Carder, J.-P. R. Wells, J. W. Cockburn, M. S. Skolnick, M. J. Steer, and M. Hopkinson, Phys. Rev. B 70, 161305 (2004).

${ }^{29}$ E. Gallardo, L. J. Martinez, A. K. Nowak, D. Sarkar, H. P. van der Meulen, J. M. Calleja, C. Tejedor, I. Prieto, D. Granados, A. G. Taboada, J. M. Garcia, and P. A. Postigo, Phys. Rev. B 81, 193301 (2010).

${ }^{30}$ R. Hanbury Brown and R. Q. Twiss, Nature (London) 178, 1046 (1956). 